\title{
NÃO-PREFERÊNCIA PARA OVIPOSIÇÃO E ALIMENTAÇÃO DE SPODOPTERA FRUGIPERDA (J. E. SMITH, 1797) (LEPIDOPTERA: NOCTUIDAE) POR CULTIVARES DE AMENDOIM
}

\section{A.P. Campos, A.L. Boiça Junior, Z.A. Ribeiro}

UniversidadeEstadual Paulista, Faculdade deCiências Agráriase Veterinárias, Departamento de Fitossanidade, Laboratório de Resistência de Plantas a Insetos, Via de Acesso Prof. Paulo Donato Castellane s/no , CEP 14884900, Jaboticabal, SP, Brasil. E-mail: apianoscki@yahoo.com.br

\section{RESUMO}

\begin{abstract}
Este trabalho buscou selecionar cultivares de amendoim resistentes a Spodoptera frugiperda, dos tipos não-preferência para oviposição e alimentação, em testes com e sem chance de escolha. Utilizaram-se cultivares de amendoim de hábitos de crescimento ereto (IAC 5, IAC 8112, IAC 22 e IAC Tatu ST) e rasteiro (IAC 503, IAC 505, IAC 147, IAC 125, IAC Caiapó e IAC Runner 886). Os testes de não-preferência para oviposição foram realizados com adultos recém-emergidos, os quais foram alimentados com solução de mel a 10\%, permanecendo nas gaiolas por quatro dias até o início das avaliações. Estas consistiram na quantificação do número de ovos e posturas, com posterior cálculo do número de ovos por postura. Para os testes de não-preferência para alimentação foram colocados discos foliares das cultivares em placas de Petri, liberando uma lagarta de terceiro ínstar por cultivar. Avaliou-se a atratividade a 1, 5, 10, 15, 30, 60, 120, 360, 720; 1.440 minutos, contando-se o número de lagartas que se alimentavam nas cultivares e, ao término do experimento, quantificou-se a área foliar consumida por cultivar. Nos testes de não-preferência para oviposição ou alimentação, com ou sem chance, nenhuma cultivar de hábitos de crescimento ereto ou rasteiro influenciou a oviposição ou alimentação de $S$. frugiperda. Dessa maneira, as cultivares de amendoim não apresentaram resistência dos tipos não-preferência para oviposição e alimentação.
\end{abstract}

PALAVRAS-CHAVE: Lagarta-do-cartucho, Arachis hypogaea, resistência de plantas, tipos de resistência.

\section{ABSTRACT}

NON-OVIPOSITIONANDNON-FEEDINGPREFERENCEOF SPODOPTERAFRUGIPERDA(J.E. SMITH, 1797) (LEPIDOPTERA: NOCTUIDAE) FOR PEANUT CULTIVARS. This study aimed to select peanut cultivars resistant to Spodoptera frugiperda, selecting non-oviposition and non-feeding preference in choice and no-choice tests. Peanut cultivars with upright growth habit (IAC 5, IAC 8112, IAC 22 and IAC TatuST) and runner growth habit (IAC 503, IAC 505, IAC 147, IAC 125, IAC Caiapó and IAC Runner 886) were evaluated. Tests of non-oviposition preference were conducted with newly emerged adults. Adults were fed a 10\% honey solution, remaining in cages for 4 days until the start of the evaluations. The evaluations consisted of quantifying the number of eggs and egg masses, followed by calculating the number of eggs per egg mass. To perform non-feeding preference tests, leaf discs from different peanut cultivars were placed in disposable Petri dishes followed by releasing of a third-instar caterpillar for each cultivar. The attractiveness at 1, 5; 10, 15, 30, 60, 120, 360, 720 and 1,440 minutes was evaluated by counting the caterpillars that were feeding on cultivars. The leaf area consumed was evaluated at the end of the experiment for each cultivar. In the tests of non-oviposition and non-feeding preference, with choice or no-choice, none of the cultivars of the upright growth and runner growth habit influenced the oviposition or food of $S$. frugiperda. Thus, the peanut cultivars showed no resistance of the non-feeding and non-oviposition types.

KEY WORDS: Fall armyworm, Arachis hypogaea, host plant resistance, types of resistance.

\section{INTRODUÇÃO}

O Estado de São Paulo destaca-se como um dos maiores produtores de amendoim do país e da região
Sudeste,comaproximadamente173mil toneladas(safra 2006/2007)(Agrianual,2008).OsmunicípiosdeDumont, JaboticabaleRibeirãoPreto são responsáveis por $89 \%$ da produção nacional de amendoim (NEGRINI, 2000). 
O amendoim, no Estado de São Paulo, é utilizado em sucessão ao plantio de cana-de-açúcar (INSTituTo de Economia Agrícola, 1999), sendo produzido em áreas de renovação de canaviais, o que proporciona uma renda alternativa de entressafra (JORGE, 1993).

Um dos grandes obstáculos à produtividade na cultura do amendoim são as pragas, dentre elas Enneothripsflavens Moulton(Thysanoptera:Thripidae), Stegastabosquella(Chambers)(Lepidoptera:Gelechiidae) eAnticarsiagemmatalisHübner(Lepidoptera:Noctuidae) (GAlLo et al., 2002), porém Spodoptera frugiperda (J. E. Smith) (Lepidoptera: Noctuidae), praga polífaga (LEIDERMAN;SAUER,1953;CruZetal.,1999), vemocorrendo de maneira acentuada nesta cultura.

Cruz et al. (1962) destacaram S. frugiperda como praga importante da parte aérea do amendoim que, dependendo da infestação, pode provocar sérios prejuízos à cultura, devorando folhas e hastes tenras das plantas. Neste sentido, SouZA; ReIs (1981) também relataram este inseto como praga desta cultura.

Vários são os casos de resistência de pragas a inseticidas, oque tambéméobservadoem $S$. frugiperda (Diez-Rodriguez; OMOto, 2001; Morillo; Notz, 2001), uma vezqueseu controlese dá praticamente comouso de produtos químicos (Costa et al., 2005; Busato et al., 2006). Assim, a resistência de plantas a insetos é uma alternativa no controle de pragas e pode ser utilizada com outras táticas de controle, diminuindo a população das pragas e minimizando os efeitos adversos de produtos químicos no meio ambiente (LARA, 1991).

Há diversos trabalhos na literatura que buscam identificar fontes de resistência dos tipos não-preferência a diversas pragas (LARA et al.,1999; BALDIN et al., 2000; Lima et al. 2002), principalmente quando se refereà não-preferência de $S$. frugiperda por genótipos de milho (SiLVeIra et al., 1998; BoIÇA Junior et al., 2001).

No entanto, na cultura do amendoim, estudos de interações entre plantas e seus herbívoros são pouco realizados, principalmente em se tratando da lagartado-cartucho. O que se verifica na literatura são estudos que buscam materiais resistentes ao tripes (BorçA JunIOR et al., 2004; MORAEs et al., 2005; LOURENÇão et al., 2007; Chagas FilHo et al., 2008) e, mais recentemente, a A. gemmatalis (BOIÇA Junior et al., 2008).

Dessa maneira, objetivou-se, neste trabalho, selecionar cultivares de amendoim, de hábitos de crescimento ereto e rasteiro, resistentes a S. frugiperda, identificando os tipos de resistência envolvidos, nãopreferência para oviposição e alimentação, em testes com e sem chance de escolha.

\section{MATERIALEMÉTODOS}

Os experimentos foram conduzidos nas dependências do Laboratório Resistência de Plantas a Inse- tos do Departamento de Fitossanidade da FCAV, UNESP, Campus de Jaboticabal, SP, sob condições controladas de temperatura $\left(25 \pm 2^{\circ} \mathrm{C}\right)$, umidade relativa ( $60 \pm 10 \%$ ) e fotofase (12 horas).

Avaliaram-se as cultivares de hábitos de crescimento ereto (IAC 5, IAC 8112, IAC 22 e IAC Tatu ST) e de crescimento rasteiro (IAC 503, IAC 505, IAC 147, IAC 125, IAC Caiapó e IAC Runner 886), as quais as sementes foram adquiridas do Instituto Agronômico de Campinas (IAC).

Foram realizados testes com e sem chance de escolha tanto para oviposição quanto para alimentação, sendo as cultivares avaliadas separadamente para cada hábito de crescimento.

As lagartas e mariposas utilizadas nos ensaios foram obtidas por meio da criação estoque, mantida em laboratório, em meio artificial à base de feijão (KASTEN JUNIOR et al., 1978).

Testes de não-preferência para oviposição de Spodoptera frugiperda, com e sem chance de escolha

As cultivares foram semeadas em copos de 500 $\mathrm{mL}$, contendo duas partes de solo e uma parte de esterco bovino, e mantidas em casa-de-vegetação. Após 20 dias da emergência, as plantas (com aproximadamente $30 \mathrm{~cm}$ de altura) foram utilizadas nos testes.

Para os testes de não-preferência para oviposição, com chance de escolha, utilizaram-se gaiolas de $70 \mathrm{~cm}$ x $60 \mathrm{~cm}$ debasee $40 \mathrm{~cm}$ dealtura, recobertas com tecido "voile". As cultivares foram dispostas dentro da gaiola de maneira equidistantes ao centro, onde foram liberados dois casais de mariposas recém-emergidas por cultivar. Os insetos permaneceram por quatro dias até a primeira avaliação e, após três dias da primeira avaliação, realizou-se a segunda avaliação. Estas consistiram na quantificação do número de ovos e de posturas, com posterior cálculo do número de ovos por postura.

Nos testes sem chance de escolha, utilizaram-se gaiolas confeccionadas a partir de garrafas plásticas (PET), as quais foram cortadas no gargalo, de modo que o copo com a planta ultrapassou o corte feito, sendo o próprio copo a base da gaiola. Cortou-se também o fundo da garrafa, sendo este a parte superior da gaiola, a qual foi coberta com tecido "voile" para evitar fugas (Fig. 1). Foram liberados dois casais de mariposas recém-emergidas por cultivar, permanecendo quatro dias até a avaliação, a qual consistiu na quantificação do número de ovos e de posturas, composterior cálculo donúmero deovos por postura.

Os adultos foram alimentados com solução de mel a $10 \%$ em ambos os testes. O alimento foi substituído diariamente e as plantas molhadas a cada dois dias com aproximadamente $25 \mathrm{~mL}$ de água. 


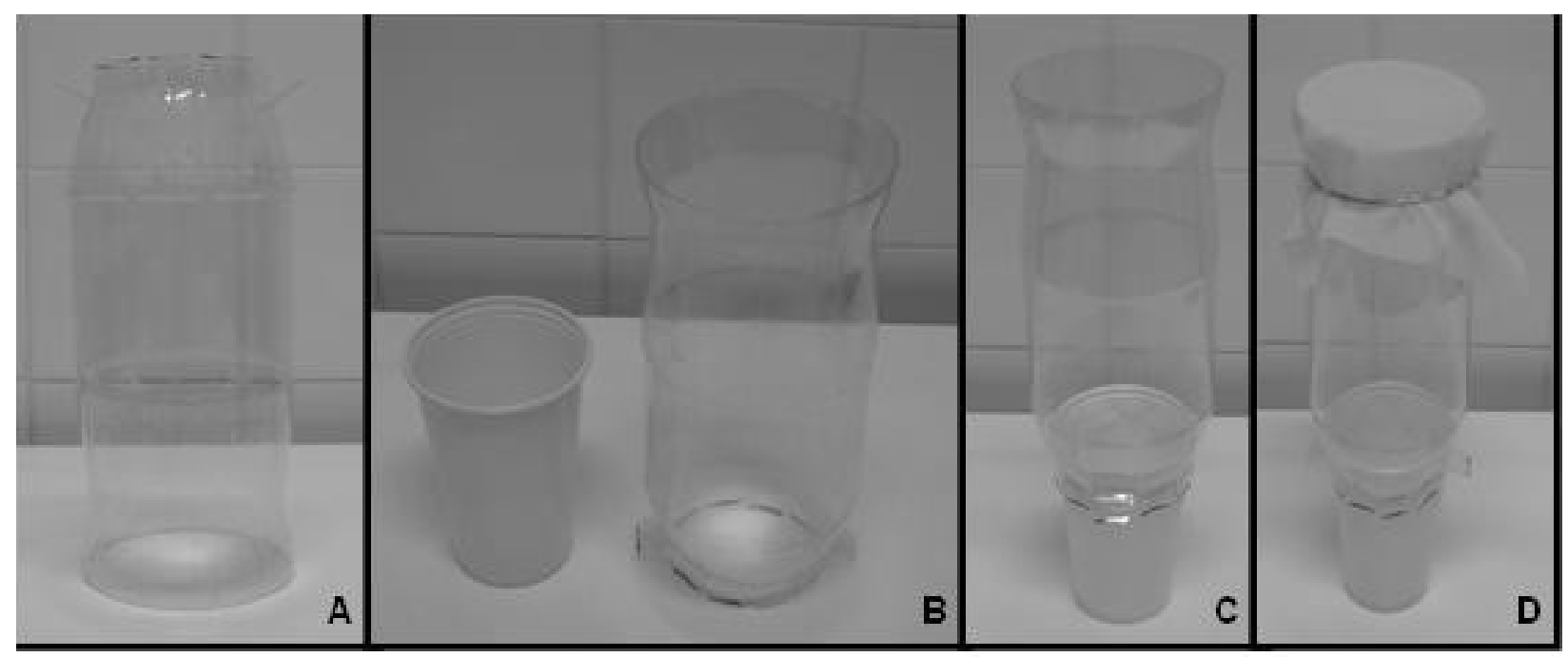

Fig. 1 - Gaiola utilizada no teste de não-preferência para oviposição sem chance de escolha, confeccionada com garrafa plástica (PET). Corte do gargalo e do fundo da garrafa (A); Copo utilizado como base da gaiola e garrafa na posição invertida (B); Gaiola pré-montada, mostrando o copo como base (C); Gaiola finalizada, com a base (copo) e a parte superior coberta com tecido "voile" (D).

Os delineamentos utilizados foram o deblocos ao acaso, com cinco repetições, para o teste com chance de escolha e inteiramente casualizado, com cinco repetições, para o teste sem chance de escolha. Os tratamentos, emambosos testes, foram quatrocultivares de hábito de crescimento ereto e seis cultivares de hábito de crescimento rasteiro.

Os dados foram submetidos à análise de variância pelo teste $\mathrm{F}$ e as médias comparadas pelo teste de Tukey, a 5\% de probabilidade.

Testes de não-preferência para alimentação de Spodoptera frugiperda, com e sem chance de escolha

Foram retirados discos foliares de 2,5 cm de diâmetro com o auxílio de um vazador. Estes discos, nos testes com chance de escolha, foram distribuídos de maneira equidistante ao centro, em placas de Petride $15 \mathrm{~cm}$ de diâmetroe $2 \mathrm{~cm}$ de altura, forradas com papel filtro umedecido. Ao centro das placas de Petri, foi liberada uma lagarta de terceiro ínstar por cultivar.

As condições para o teste sem chance de escolha foram semelhantes ao descrito anteriormente, porém utilizaram-se placas de Petri de $8,5 \mathrm{~cm}$ de diâmetro e $1,5 \mathrm{~cm}$ de altura, com apenas um disco foliar da cultivar por placa, liberando-se no interior uma lagarta de terceiro ínstar.

A avaliação em ambos os testes consistiu-se na contagem do número de lagartas atraídas para os discos foliares e na quantificação do consumo foliar ao final do experimento. Quanto à atratividade, as avaliações foram realizadas a 1, 5, 10, 15, 30, 60, 120, 360, 720, 1.440 minutos após a liberação das lagartas.
Para medir a área foliar de cada disco ofertado às lagartas, utilizou-se o aparelho LICOR LI-3000A. O consumo foi calculado através da diferença entre a alíquota $\left(3,23 \mathrm{~cm}^{2}\right)$ do disco foliar fornecido no início dos testes e as porções restantes após o consumo das lagartas.

Os delineamentos utilizados foram o de blocos ao acaso, com 13 repetições, para o teste com chance de escolha e inteiramente casualizado, com 13 repetições, para o teste sem chance de escolha. Os tratamentos, em ambos os testes, foram quatro cultivares de hábito de crescimento ereto e seis cultivares de hábito de crescimento rasteiro.

Os dados foram submetidos à análise de variância pelo teste $\mathrm{F}$ e as médias comparadas pelo teste de Tukey, a $5 \%$ de probabilidade.

\section{RESULTADOSEDISCUSSÃO}

Testes de não-preferência para oviposição de Spodoptera frugiperda, com e sem chance de escolha

No teste com chance de escolha para oviposição (Tabela 1), não houve diferença quanto ao número de ovos, posturas e ovos por postura entre os tratamentos. Nota-se, contudo, entre as cultivares de hábito de crescimento ereto, queIAC TatuST tende a ser menos preferido se comparado IAC 8112 equeas cultivares IAC 5 e IAC 22 apresentam valores intermediários quanto a preferência do inseto. Dessa mesma forma, entre as cultivares de hábito de crescimento rasteiro, não houve diferença significativa entre as cultivares, porém nota-se que IAC Runner 886 proporcionou 
menores valores médios de ovos, posturas e ovos por postura, seguida das cultivares IAC 503 e IAC 125, tendendo a ser menos preferidas em comparaçãocom IAC 505, a qual proporcionou os maiores valores nos parâmetros avaliados.

O mesmo ocorreu para o teste sem chance de escolha (Tabela 2), em que não foram detectadas diferenças entre os tratamentos quanto ao número de ovos, posturas e ovos por postura. Contudo, quando se isola o inseto, não dando a chance desse escolher qual material prefere ovipositar, nota-se uma ligeira inversão nos dados, ou seja, a cultivar IAC 503, de hábito de crescimento rasteiro, foi a que apresentou uma tendência de maiores valores no teste sem chance de escolha, sendo as cultivares IAC Runner 886, IAC Caiapó, IAC 125 e IAC 505 as que mostraram uma menor oviposição nos parâmetros avaliados.

Ensaios envolvendo a fertilidade da espécie, com quantificação de ovos e/ou posturas possui grande variação, afinal cada indivíduo é único, havendo fêmeas com alta capacidade reprodutiva eoutras não, mesmo sendo criadas da mesma forma. Diante disso, ocorre uma grande variação na quantidade de ovos, posturas e ovos por postura, sendo assim, essa variação pode ser detectada na estatística, evidenciados nos coeficientes de variação (Tabela 2).

LARA et al. (1999), estudando a preferência para oviposição de Alabama argillacea (Huebner, 1818) (Lepidoptera: Noctuidae) por genótipos de algodoei- ro, evidenciaram resultados similares, ou seja, não apresentaram diferenças significativas quanto ao número de ovos nos diferentes genótipos testados e concluíram que os genótipos não influenciaram o comportamento de oviposição desse inseto.

Em testes denão-preferência para oviposiçãode $S$. frugiperda por genótipos de sorgo, LORDELLO; LARA (1980) concluíram quehouve uma marcante preferência para a oviposição na face inferior das folhas se comparados com a face superior, porém não detectaram diferenças significativas entre os genótipos testados quanto ao número de ovos, posturas e ovos por postura.

Ng et al. (1990), nos Estados Unidos da América, ao testarem híbridos de milho considerados resistentes e suscetíveis a S. furgiperda, evidenciaram maior preferência para a oviposição em um híbrido suscetível (SC229 x Tx601); além disso, houve maior quantidade de ovos na face inferior das folhas, reforçando essa marcante preferência.

Recentemente, no Brasil, pesquisas envolvendo genótipos de milho obtidos pelo melhoramentogenético convencional são escassas, sendo comumente pesquisados genótipos geneticamente modificados. WAQUIL et al. (2002) e FERNANDES et al. (2003), ao trabalharem com milho Bt, comentam que esses materiais proporcionam uma menor densidade populacionale danos de $S$. frugiperda, evidenciando esse direcionamento dos estudos atuais.

Tabela 1 - Número médio de ovos, posturas e ovos por postura de Spodoptera frugiperda em cultivares de amendoim, em teste com chance de escolha. Jaboticabal, SP, 2008.

\begin{tabular}{|c|c|c|c|}
\hline \multirow[t]{2}{*}{ Cultivares } & \multicolumn{3}{|c|}{ Teste com cultivares de hábito de crescimento ereto ${ }^{1}$} \\
\hline & № de ovos 2 & № de posturas ${ }^{2}$ & № de ovos/postura ${ }^{2}$ \\
\hline IAC 5 & $1.069,40 \mathrm{a}$ & 4,80 a & 185,23 a \\
\hline IAC 8112 & $1.221,40 \mathrm{a}$ & $6,20 \mathrm{a}$ & 212,26 a \\
\hline IAC 22 & $1.042,00 \mathrm{a}$ & $4,40 \mathrm{a}$ & 211,83 a \\
\hline IAC Tatu ST & 797,20 a & $4,00 \mathrm{a}$ & 216,81 a \\
\hline F (tratamento) & $0,43^{\text {ns }}$ & $0,64^{\text {ns }}$ & $0,50^{\text {ns }}$ \\
\hline \multirow[t]{2}{*}{ C.V. $(\%)$} & 36,12 & 22,78 & 29,19 \\
\hline & \multicolumn{3}{|c|}{ Teste com cultivares de hábito de crescimento rasteiro ${ }^{1}$} \\
\hline IAC 503 & $737,40 \mathrm{a}$ & $5,00 \mathrm{a}$ & 147,69 a \\
\hline IAC 505 & $1.696,00 \mathrm{a}$ & $8,60 \mathrm{a}$ & $180,22 \mathrm{a}$ \\
\hline IAC 147 & $1.044,40 \mathrm{a}$ & $6,20 \mathrm{a}$ & $166,51 \mathrm{a}$ \\
\hline IAC 125 & $929,00 \mathrm{a}$ & $5,40 \mathrm{a}$ & 180,60 a \\
\hline IAC Caiapó & $1.365,60 \mathrm{a}$ & 7,60 a & 183,41 a \\
\hline IAC Runner 886 & 579,20 a & 3,80 a & 138,29 a \\
\hline F (tratamento) & $2,06^{\text {ns }}$ & $1,97^{\mathrm{ns}}$ & $0,84^{\mathrm{ns}}$ \\
\hline C.V. $(\%)$ & 30,99 & 19,94 & 15,19 \\
\hline
\end{tabular}

${ }^{1}$ Médias seguidas pela mesma letra na coluna não diferem significativamente entre si pelo teste de Tukey, a 5\% de probabilidade.

${ }^{2}$ Para análise os dados foram transformados em $(x+1,0)^{1 / 2}$. 
Ainda entre os noctuídeos, porém em amendoim, BOIÇAJUNIOR etal. (2008), trabalhando com A. gemmatalis em testes de não-preferência para oviposição, sem chance de escolha, mostraram a preferência desse inseto pelo genótipo 'IAC 147' em relação ao 'IAC 8112'; além disso, esse material também foi o menos preferido para alimentação nos testes com e sem chance de escolha.

Testes de não-preferência para alimentação de Spodoptera frugiperda, com e sem chance de escolha

A não-preferência para alimentação com chance de escolha (Tabela 3), no que se refere à atratividade e ao consumo foliar pelas lagartas, não sofreu influência das cultivares, ou seja, não houve diferença significativa em nenhuma das avaliações. Contudo, dentre as cultivares dehábito de crescimento rasteiro, aos 1.440 minutos após a liberação das lagartas, notase uma quantidade maior de lagartas se alimentando de IAC 125 quando comparado a IAC 503.

Observando os tempos das avaliações (Tabela 3), nota-se tendência de aumento gradativo no número de lagartas atraídas pelas cultivares, porém essa não é uma constância, o que pode ser explicado pelo fato de haver mais de uma cultivar nas placas, liberando voláteis atrativos de alimentação que, de acordo com
LARA (1991), é a primeira fase da seleção hospedeira para alimentação ou oviposição de um inseto fitófago, que possibilita a movimentação na direção e entre os discos foliares. Além disso, pode ter ocorrido influência pelo comportamento canibal da espécie, o que proporcionou a permanência de uma lagarta em cada disco foliar.

Nos testes sem chance de escolha (Tabela 4), tanto para as cultivares de hábito de crescimento ereto quanto para as de crescimento rasteiro, no que se refere à atratividade e ao consumo foliar das lagartas pelas cultivares, não houve diferenças significativas.

No decorrer dos tempos das avaliações (Tabela4), observam-se aumento gradativo na quantidade de lagartas atraídas pelas cultivares e uma constância na permanência no disco foliar. Isso ocorre, provavelmente, por não existirem outras cultivares liberando voláteis atrativos de alimentação, e assim os insetos locomovem-se para o alimento e permanecem se alimentando.

BoiçA Junior et al. (2001), buscando genótipos de milho resistentes a $S$. frugiperda em testes de nãopreferência para alimentação, também não encontraram diferenças quanto à atratividade dos genótipos sobre as lagartas, porém, quanto à matéria seca consumida, 'AG 8012' foi o mais consumido, diferindo de 'C 909' e 'C 511'.

Tabela 2 - Número médio de ovos, posturas e ovos por postura de Spodoptera frugiperda em cultivares de amendoim, em teste sem chance de escolha. Jaboticabal, SP, 2008.

\begin{tabular}{|c|c|c|c|}
\hline \multirow[t]{2}{*}{ Cultivares } & \multicolumn{3}{|c|}{ Teste com cultivares de hábito de crescimento ereto ${ }^{1}$} \\
\hline & $\mathrm{N}^{o}$ de ovos ${ }^{2}$ & № de posturas ${ }^{2}$ & № de ovos/ postura ${ }^{2}$ \\
\hline IAC 5 & $54,00 \mathrm{a}$ & $0,60 \mathrm{a}$ & $18,00 \mathrm{a}$ \\
\hline IAC 8112 & $252,00 \mathrm{a}$ & $2,20 \mathrm{a}$ & $41,20 \mathrm{a}$ \\
\hline IAC 22 & $-*$ & $-*$ & $-*$ \\
\hline IAC Tatu ST & $-*$ & $-*$ & $-*$ \\
\hline F (tratamento) & $1,55^{\mathrm{ns}}$ & $1,76^{\text {ns }}$ & $1,43^{\text {ns }}$ \\
\hline \multirow[t]{2}{*}{ C.V. $(\%)$} & 90,07 & 40,46 & 73,84 \\
\hline & \multicolumn{3}{|c|}{ Teste com cultivares de hábito de crescimento rasteiro ${ }^{1}$} \\
\hline IAC 503 & $1.067,20 \mathrm{a}$ & $8,00 \mathrm{a}$ & 106,47 a \\
\hline IAC 505 & 386,60 a & $4,60 \mathrm{a}$ & 92,83 a \\
\hline IAC 147 & 751,40 a & $4,80 \mathrm{a}$ & 126,43 a \\
\hline IAC 125 & $357,40 \mathrm{a}$ & $3,20 \mathrm{a}$ & 87,47 a \\
\hline IAC Caiapó & $309,80 \mathrm{a}$ & $2,60 \mathrm{a}$ & 79,37 a \\
\hline IAC Runner 886 & $324,50 \mathrm{a}$ & $2,00 \mathrm{a}$ & 83,69 a \\
\hline F (tratamento) & $1,03^{\text {ns }}$ & $1,62^{\mathrm{ns}}$ & $0,49^{\mathrm{ns}}$ \\
\hline C.V. $(\%)$ & 66,51 & 32,92 & 50,44 \\
\hline
\end{tabular}

${ }^{1}$ Médias seguidas pela mesma letra na coluna não diferem significativamente entre si pelo teste de Tukey, a $5 \%$ de probabilidade.

2Para análise os dados foram transformados em $(x+1,0)^{1 / 2}$.

*Dados não analisados devido a ausência de posturas. 
Tabela 3 - Número de lagartas de Spodoptera frugiperda atraídas por cultivares de amendoim, em diferentes intervalos de tempo após a liberação, e área foliar consumida, em teste com chance de escolha. Jaboticabal, SP, 2008.

\begin{tabular}{|c|c|c|c|c|c|c|c|c|c|c|c|}
\hline \multirow[t]{3}{*}{ Cultivares } & \multicolumn{10}{|c|}{ Teste com cultivares de hábito de crescimento ereto ${ }^{1}$} & \multirow{2}{*}{$\begin{array}{l}\text { Área foliar } \\
\text { consumida } \\
\left(\mathrm{cm}^{2}\right)\end{array}$} \\
\hline & \multicolumn{10}{|c|}{ Tempo (minutos) após a liberação das lagartas ${ }^{2}$} & \\
\hline & $1^{\prime}$ & $5^{\prime}$ & $10^{\prime}$ & $15^{\prime}$ & $30^{\prime}$ & $60^{\prime}$ & $120^{\prime}$ & $360^{\prime}$ & 720' & $1.440^{\prime}$ & \\
\hline IAC 5 & $0,61 \mathrm{a}$ & $0,69 \mathrm{a}$ & $1,00 \mathrm{a}$ & $1,08 \mathrm{a}$ & $1,08 \mathrm{a}$ & $1,23 \mathrm{a}$ & $1,23 \mathrm{a}$ & $1,08 \mathrm{a}$ & $0,85 \mathrm{a}$ & $0,92 \mathrm{a}$ & $0,80 \mathrm{a}$ \\
\hline IAC 8112 & $0,46 \mathrm{a}$ & $0,61 \mathrm{a}$ & $0,69 a$ & $0,69 a$ & $0,69 \mathrm{a}$ & $0,61 \mathrm{a}$ & $0,61 \mathrm{a}$ & $0,54 \mathrm{a}$ & $0,85 \mathrm{a}$ & $1,08 \mathrm{a}$ & $0,66 \mathrm{a}$ \\
\hline IAC 22 & $0,77 \mathrm{a}$ & $0,92 \mathrm{a}$ & $1,00 \mathrm{a}$ & $1,08 \mathrm{a}$ & $1,08 \mathrm{a}$ & $1,08 \mathrm{a}$ & $1,08 \mathrm{a}$ & $1,15 \mathrm{a}$ & $1,15 \mathrm{a}$ & $0,92 \mathrm{a}$ & $0,82 \mathrm{a}$ \\
\hline IAC Tatu ST & $0,69 \mathrm{a}$ & $0,92 \mathrm{a}$ & $0,92 \mathrm{a}$ & $0,92 \mathrm{a}$ & $0,92 \mathrm{a}$ & $0,92 \mathrm{a}$ & $0,76 \mathrm{a}$ & $0,92 \mathrm{a}$ & $0,92 \mathrm{a}$ & $1,08 \mathrm{a}$ & $1,00 \mathrm{a}$ \\
\hline F (tratamento) & $0,36^{\mathrm{ns}}$ & $0,57^{\text {ns }}$ & $0,25^{\mathrm{ns}}$ & $0,44^{\mathrm{ns}}$ & $0,44^{\text {ns }}$ & $0,89^{\text {ns }}$ & $1,30^{\text {ns }}$ & $1,72^{\mathrm{ns}}$ & $0,48^{\mathrm{ns}}$ & $0,15^{\text {ns }}$ & $0,50^{\text {ns }}$ \\
\hline C.V. $(\%)$ & 24,70 & 24,16 & 26,75 & 25,57 & 25,57 & 25,97 & 23,84 & 20,87 & 18,97 & 18,84 & 24,78 \\
\hline \multicolumn{12}{|c|}{ Teste com cultivares de hábito de crescimento rasteiro ${ }^{1}$} \\
\hline IAC 503 & $0,42 \mathrm{a}$ & $0,58 \mathrm{a}$ & $0,58 \mathrm{a}$ & $0,83 \mathrm{a}$ & $0,75 \mathrm{a}$ & $0,67 \mathrm{a}$ & $0,83 \mathrm{a}$ & $0,75 \mathrm{a}$ & $0,58 \mathrm{a}$ & $0,42 \mathrm{~b}$ & $1,25 \mathrm{a}$ \\
\hline IAC 505 & $0,58 \mathrm{a}$ & $0,42 \mathrm{a}$ & $0,50 \mathrm{a}$ & $0,67 \mathrm{a}$ & $0,67 \mathrm{a}$ & $0,67 \mathrm{a}$ & $0,42 \mathrm{a}$ & $0,67 \mathrm{a}$ & $0,67 \mathrm{a}$ & $0,58 \mathrm{ab}$ & $1,09 \mathrm{a}$ \\
\hline IAC 147 & $1,25 \mathrm{a}$ & $1,17 \mathrm{a}$ & $0,67 \mathrm{a}$ & $0,67 \mathrm{a}$ & $0,92 \mathrm{a}$ & $0,67 \mathrm{a}$ & $0,83 \mathrm{a}$ & $0,75 \mathrm{a}$ & $0,75 \mathrm{a}$ & $0,50 \mathrm{ab}$ & $1,11 \mathrm{a}$ \\
\hline IAC 125 & $0,50 \mathrm{a}$ & $0,67 \mathrm{a}$ & $0,83 \mathrm{a}$ & $0,67 \mathrm{a}$ & $0,58 \mathrm{a}$ & $0,58 \mathrm{a}$ & $1,17 \mathrm{a}$ & $1,08 \mathrm{a}$ & $1,00 \mathrm{a}$ & $1,25 \mathrm{a}$ & $1,42 \mathrm{a}$ \\
\hline IAC Caiapó & $0,92 \mathrm{a}$ & $0,83 \mathrm{a}$ & $0,67 \mathrm{a}$ & $0,75 \mathrm{a}$ & $0,67 \mathrm{a}$ & $0,67 \mathrm{a}$ & $1,00 \mathrm{a}$ & $0,92 \mathrm{a}$ & $1,25 \mathrm{a}$ & $0,68 \mathrm{ab}$ & $1,44 \mathrm{a}$ \\
\hline IAC Runner 886 & $0,67 \mathrm{a}$ & $0,75 \mathrm{a}$ & $0,58 \mathrm{a}$ & $0,33 \mathrm{a}$ & $0,50 \mathrm{a}$ & $0,83 \mathrm{a}$ & $0,92 \mathrm{a}$ & $1,00 \mathrm{a}$ & 0,75 a & $0,83 a b$ & $1,42 \mathrm{a}$ \\
\hline F (tratamento) & $1,75^{\mathrm{ns}}$ & $0,84^{\mathrm{ns}}$ & $0,30^{\mathrm{ns}}$ & $0,60^{\mathrm{ns}}$ & $0,37^{\mathrm{ns}}$ & $0,07^{\mathrm{ns}}$ & $1,02^{\text {ns }}$ & $1,02^{\mathrm{ns}}$ & $1,39^{\mathrm{ns}}$ & $2,54^{*}$ & $1,17^{\mathrm{ns}}$ \\
\hline C.V. $(\%)$ & 23,32 & 25,31 & 22,53 & 23,95 & 21,69 & 22,93 & 23,14 & 17,75 & 21,25 & 19,69 & 13,09 \\
\hline
\end{tabular}

${ }^{1}$ Médias seguidas pela mesma letra na coluna não diferem significativamente entre si pelo teste de Tukey, a $5 \%$ de probabilidade.

${ }^{2}$ Para análise os dados foram transformados em $(x+1,0)^{1 / 2}$.

${ }^{3}$ Para análise os dados foram transformados em $(x+0,5)^{1 / 2}$.

Tabela 4 - Número de lagartas de Spodoptera frugiperda atraídas por cultivares de amendoim, em diferentes intervalos de tempo após a liberação, e área foliar consumida, em teste sem chance de escolha. Jaboticabal, SP, 2008.

\begin{tabular}{|c|c|c|c|c|c|c|c|c|c|c|c|}
\hline \multirow[t]{3}{*}{ Cultivares } & \multicolumn{10}{|c|}{ Teste com cultivares de hábito de crescimento ereto ${ }^{1}$} & \multirow{2}{*}{$\begin{array}{l}\text { Área foliar } \\
\text { consumida } \\
\quad\left(\mathrm{cm}^{2}\right)\end{array}$} \\
\hline & \multicolumn{10}{|c|}{ Tempo (minutos) após a liberação das lagartas² } & \\
\hline & $1^{\prime}$ & $5^{\prime}$ & $10^{\prime}$ & $15^{\prime}$ & $30^{\prime}$ & $60^{\prime}$ & $120^{\prime}$ & $360^{\prime}$ & $720^{\prime}$ & $1440^{\prime}$ & \\
\hline IAC 5 & 0,46 a & $0,54 \mathrm{a}$ & $0,54 \mathrm{a}$ & $0,54 \mathrm{a}$ & $0,77 \mathrm{a}$ & $0,77 \mathrm{a}$ & $0,77 \mathrm{a}$ & $0,85 \mathrm{a}$ & $0,85 \mathrm{a}$ & $1,00 \mathrm{a}$ & 1,36 a \\
\hline IAC 8112 & 0,38 a & 0,38 a & $0,38 \mathrm{a}$ & $0,38 \mathrm{a}$ & $0,61 \mathrm{a}$ & $0,85 \mathrm{a}$ & $0,92 \mathrm{a}$ & $1,00 \mathrm{a}$ & $1,00 \mathrm{a}$ & $1,00 \mathrm{a}$ & $1,28 \mathrm{a}$ \\
\hline IAC 22 & $0,15 \mathrm{a}$ & $0,15 \mathrm{a}$ & $0,23 \mathrm{a}$ & $0,23 \mathrm{a}$ & $0,38 \mathrm{a}$ & $0,54 \mathrm{a}$ & $0,92 \mathrm{a}$ & $1,00 \mathrm{a}$ & $1,00 \mathrm{a}$ & $1,00 \mathrm{a}$ & $1,68 \mathrm{a}$ \\
\hline IAC Tatu ST & $0,08 \mathrm{a}$ & 0,23 a & $0,23 \mathrm{a}$ & $0,23 \mathrm{a}$ & $0,38 \mathrm{a}$ & $0,85 \mathrm{a}$ & $0,92 \mathrm{a}$ & $1,00 \mathrm{a}$ & $1,00 \mathrm{a}$ & $1,00 \mathrm{a}$ & $1,42 \mathrm{a}$ \\
\hline F (tratamento) & $2,34^{\text {ns }}$ & $1,76^{\mathrm{ns}}$ & $1,24^{\text {ns }}$ & $1,24^{\mathrm{ns}}$ & $1,92^{\mathrm{ns}}$ & $1,48^{\text {ns }}$ & $0,73^{\text {ns }}$ & $2,18^{\text {ns }}$ & $2,18^{\mathrm{ns}}$ & $0,00^{\text {ns }}$ & $0,93^{\text {ns }}$ \\
\hline C.V. $(\%)$ & 16,07 & 16,91 & 17,28 & 17,28 & 16,60 & 13,63 & 9,86 & 5,56 & 5,56 & 0,00 & 17,66 \\
\hline \multicolumn{12}{|c|}{ 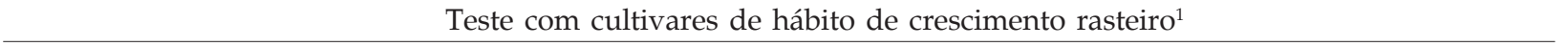 } \\
\hline IAC 503 & 0,15 a & $0,31 \mathrm{a}$ & $0,31 \mathrm{a}$ & $0,31 \mathrm{a}$ & $0,46 \mathrm{a}$ & $1,00 \mathrm{a}$ & $0,85 \mathrm{a}$ & $0,92 \mathrm{a}$ & $0,92 \mathrm{a}$ & $0,92 \mathrm{a}$ & 1,53 a \\
\hline IAC 505 & 0,08 a & 0,15 a & $0,15 \mathrm{a}$ & $0,23 \mathrm{a}$ & $0,38 \mathrm{a}$ & $0,69 \mathrm{a}$ & 0,69 a & 0,77 a & 0,85 a & 0,77 a & $1,69 \mathrm{a}$ \\
\hline IAC 147 & 0,31 a & 0,38 a & $0,38 \mathrm{a}$ & $0,46 \mathrm{a}$ & $0,69 \mathrm{a}$ & $0,69 \mathrm{a}$ & 0,77 a & $0,85 \mathrm{a}$ & 0,85 a & $0,85 \mathrm{a}$ & $1,30 \mathrm{a}$ \\
\hline IAC 125 & $0,23 \mathrm{a}$ & 0,23 a & $0,31 \mathrm{a}$ & $0,31 \mathrm{a}$ & $0,54 \mathrm{a}$ & $0,62 \mathrm{a}$ & 0,77 a & $0,85 \mathrm{a}$ & $0,92 \mathrm{a}$ & $1,00 \mathrm{a}$ & $1,49 \mathrm{a}$ \\
\hline IAC Caiapó & $0,31 \mathrm{a}$ & $0,38 \mathrm{a}$ & $0,54 \mathrm{a}$ & 0,69 a & $0,77 \mathrm{a}$ & $0,77 \mathrm{a}$ & $0,85 \mathrm{a}$ & $0,62 \mathrm{a}$ & 0,77 a & $0,92 \mathrm{a}$ & $1,49 \mathrm{a}$ \\
\hline IAC Runner 886 & 0,23 a & $0,23 \mathrm{a}$ & $0,23 \mathrm{a}$ & $0,23 \mathrm{a}$ & $0,38 \mathrm{a}$ & $0,69 a$ & $0,69 \mathrm{a}$ & 0,77 a & 0,62 a & $0,92 \mathrm{a}$ & $1,01 \mathrm{a}$ \\
\hline F (tratamento) & $0,59^{\text {ns }}$ & $0,53^{\text {ns }}$ & $1,04^{\text {ns }}$ & $1,84^{\mathrm{ns}}$ & $1,38^{\text {ns }}$ & $1,24^{\text {ns }}$ & $0,33^{\text {ns }}$ & $0,86^{\mathrm{ns}}$ & $1,18^{\text {ns }}$ & $0,87^{\mathrm{ns}}$ & $1,67^{\mathrm{ns}}$ \\
\hline C.V. $(\%)$ & 16,00 & 17,06 & 17,15 & 17,00 & 16,79 & 13,81 & 13,62 & 12,72 & 11,87 & 9,26 & 18,40 \\
\hline
\end{tabular}

${ }^{1}$ Médias seguidas pela mesma letra na coluna não diferem significativamente entre si pelo teste de Tukey, a $5 \%$ de probabilidade.

${ }^{2}$ Para análise os dados foram transformados em $(x+1,0)^{1 / 2}$.

${ }^{3}$ Para análise os dados foram transformados em $(x+0,5)^{1 / 2}$. 
Outro trabalho que definiu a não-preferência para alimentação como fonte de resistência foi relatado por Silveira et al. (1998), que identificaram os germoplasmas Zapalote Chico e Mp707 como resistentes a lagartas de primeiro e quinto ínstares de $S$. frugiperda.

Com relação a genótipos deamendoim resistentes a A. gemmatalis, BoIçA JunIor et al. (2008) não observaram diferenciações quanto à atratividade das lagartas pelos genótipos e sim no consumo foliar, destacando-se como menos preferidos para a alimentação do inseto o 'IAC 147', 'IAC Caiapó' e 'IAC Runner 886', em testes com e sem chance de escolha.

\section{CONCLUSÃO}

As cultivares de amendoim de hábitos de crescimento ereto e rasteironão apresentam resistência dos tipos não-preferência para oviposição e alimentação a $S$. frugiperda.

\section{AGRADECIMENTOS}

Ao CNPq, pela concessão da bolsa de mestrado à primeira autora e de produtividade em pesquisa ao segundo; ao Dr. Ignácio José de Godoy, Pesquisador doInstituto Agronômico de Campinas, SP, pelo fornecimento das sementes das cultivares.

\section{REFERÊNCIAS}

AGRIANUAL 2008: Anuário de agricultura brasileira. São Paulo: FNP Consultoria \& Comércio. 2008. p.182.

BALDIN, E.L.L.; TOSCANO, L.C.; LIMA, A.C.S.; LARA, F.M.; BOIÇA JUNIOR, A.L. Preferência para oviposição de Bemisia tabaci Biótipo "B" por genótipos de Cucurbita moschata e Cucurbita maxima. Boletín de Sanidad Vegetal Plagas, v.26, p.409-413, 2000.

BOIÇA JUNIOR, A.L.; MARTINELLI, S.; PEREIRA, M.F.A. Resistência de genótipos de milho ao ataque de Spodoptera frugiperda (J.E. Smith, 1797) e Helicoverpa zea (Boddie, 1850) (Lepidoptera: Noctuidae). Ecossistema, v.26, n.1, p.86-90, 2001.

BOIÇA JUNIOR, A.L.; SANTOS, T.M.; CENTURION, M.A.P.C; JORGE, J.M. Resistência de genótipos de amendoim Arachis hypogaea L. a Enneothrips flavens Moulton, 1941 (Thysanoptera: Thripidae). Bioscience Journal, v.20, n.1, p.75-80, 2004.

BOIÇA JUNIOR, A.L.; PITTA, R.M.; JESUS, F.G.; CAMPOS, A.P. Não-preferência para alimentação e para oviposição de genótipos de amendoim a Anticarsia gemmatalis Hübner (Lepidoptera: Noctuidae). Revista de Agricultura, v.83, n.1, p.66-74, 2008.

BUSATO, G.R.; GRÜTZMACHER, A.D.; GARCIA, M.S.; ZOTTI, M.J.; NÖRBERG, S.D.; MAGALHÃES, T.R.; MAGALHÃES, J.B. Susceptibilidade de lagartas dos biótipos milho e arroz de Spodoptera frugiperda (J.E. Smith, 1797) (Lepidoptera: Noctuidae) a inseticidas com diferentes modos de ação. Ciência Rural, v.36, n.1, p.15-20, 2006.

CHAGAS FILHO, N.R.; BOIÇA JUNIOR, A.L.; GODOY J.I.; LOURENÇÃO, A.L.; RIBEIRO, Z.A. Resistência de cultivares de amendoim de hábitos de crescimento ereto a Enneothrips flavens Moulton (Thysanoptera: Thripidae). Arquivos do Instituto Biológico, São Paulo, v.75, n.n.2, p.149-156, 2008.

COSTA, M.A.G., GRÜTZMACHER, A.D.; MARTINS, J.F.S.; COSTA, E.C.; STORCH, G.; STEFANELLO JUNIOR, G.J. Eficácia de diferentes inseticidas e de volume de calda no controle de Spodoptera frugiperda na cultura de milho e sorgo cultivados em várzea. Ciência Rural, v.35, n.6, p.1234-1242, 2005.

CRUZ, B.P.B.; FIGUEIREDO, M.B.; ALMEIDA, E. Principais doenças e pragas do amendoim no Estado de São Paulo. Biológico, São Paulo, v.28, n.7, p.189-195, 1962.

CRUZ, I.; FIGUEIREDO, M.L.C.; MATOSO, M.J. Controle biológico de Spodoptera frugiperda utilizando o parasitóide de ovos Trichogramma. Sete Lagoas: Embrapa/CNPMS, 1999. 40p. (Circular Técnica Número 30).

DIEZ-RODRIGUEZ, G.I.; OMOTO, C. Herança da resistência de Spodoptera frugiperda (J.E. Smith) (Lepidoptera: Noctuidae) a lambda-cialotrina. Neotropical Entomology, v.30, n.2, p.311-316, 2001.

FERNANDES, O.D.; PARRA, J.R.P.; FERREIRA NETO, A.; PÍCOLI, R.; BORGATTO, A.F.; DEMÉTRIO, C.G.B. Efeito do milho geneticamente modificado MON810 sobre a lagarta-do-cartucho Spodoptera frugiperda (J. E. Smith, 1797) (Lepidoptera: Noctuidae). Revista Brasileira de Milho e Sorgo, v.2, n.2, p.25-35, 2003.

GALLO, D.; NAKANO, O.; SILVEIRA NETO, S.; CARVALHO, R.P.L.; BATISTA, G.C.; BATISTA FILHO, E.; PARRA, J.R.P.; ZUCCHI, R.A.; ALVES, S.B.; VENDRAMIM, J.D.; MARCHINI, L.C.; LOPES, J.R.S.; OMOTO, C. Entomologia agrícola. 10.ed. Piracicaba: FEALQ, 2002. 920p.

INSTITUTO DE ECONOMIA AGRÍCOLA. (SP). Estimativa e previsão de safras. Informações Econômicas, v.29 n.12, p.107, 1999.

JORGE, J.M. Resistência de genótipos de amendoim (Arachis hypogaea L.) ao ataque de Enneothrips flavens (Moulton, 1941) (Thysanoptera, Thripidae), na região de Jaboticabal, SP. 
Brasil. 1993. 61p. Monografia (Trabalho de Graduação em Agronomia) - Faculdade de Ciências Agrárias e Veterinárias, Universidade Estadual Paulista, Jaboticabal, 1993.

KASTEN JUNIOR, A.A.; PRECETTI, C.M.; PARRA, J.R.P. Dados biológicos comparativos de Spodoptera frugiperda em duas dietas artificiais e substrato natural. Revista de Agricultura, v.53, n.1-2, p.68-78, 1978.

LARA, F.M. Princípios de resistência de plantas a insetos. São Paulo: Ícone, 1991. 336p.

LARA, F.M.; FERREIRA, A.; CAMPOS, A.R.; SOARES, J.J. Tipos de resistência a Alabama argillacea (Huebner) (Lepidoptera: Noctuidae) envolvidos em genótipos de algodoeiro: I - Não-preferência. Anais da Sociedade Entomológica do Brasil, v.28, n.4, p.739-744, 1999.

LEIDERMAN, L.; SAUER, H.F.G. A lagarta dos milharais. Biológico, São Paulo, v.19, n.6, p.105-113, 1953.

LIMA, C.S.; LARA, F.M.; BARBOSA, J.C. Preferência para oviposição de Bemisia tabaci (Genn.) Biótipo B (Hemiptera: Aleyrodidae) em genótipos de soja, sob condições de campo. Neotropical Entomology, v.31, n.2, p.297-303, 2002.

LORDELLO, A.I.L; LARA, F.M. Preferência para oviposição de Spodoptera frugiperda (J.E. Smith, 1797) em sorgo, em condições de laboratório. Anais da Sociedade Entomológica do Brasil, v.9, n.1, p.11-21, 1980.

LOURENÇÃO, A.L.; MORAES, A.R.A.; GODOY, I.J.; AMBROSANO, G.M.B. Efeito da infestação de Enneothrips flavens Moulton sobre o desenvolvimento de cultivares de amendoim. Bragantia, v.66, n.4, p.527533, 2007.
MORAES, A.R.A.; LOURENÇÃO, A.L.; GODOY, I.J.; TEIXEIRA, G.C. Infestation by Enneothrips flavens Moulton and yield of peanut cultivars. Scientia Agricola, v.62, n.5, p.469-472, 2005.

MORILLO, F.; NOTZ, A. Resistência de Spodoptera frugiperda (Smith) (Lepidoptera: Noctuidae) a lambdacihalotrina y metomil. Entomotropica, v.16, n.2, p.79-87, 2001.

NEGRINI, C. Produtores descobrem o tipo rasteiro. $A$ Granja, v.56, n.614, p.27-30, 2000.

NG, S.S.; DAVIS, F.M.; WILLIAMS, W.P. Ovipositional response of southwestern corn borer (Lepidoptera: Pyralidae) and fall armyworm (Lepidoptera: Noctuidae) to selected maize hybrids. Journal of Economic Entomology, v.83, n.4, p.1575-1577, 1990.

SILVEIRA, L.C.P.; VENDRAMIM, J.D.; ROSSETTO, C.J. Não-preferência para alimentação da lagarta-docartucho em milho. Bragantia, v.57, n.1, p.105-111, 1998.

SOUZA, J.C.; REIS, P.R. Reconhecimento e controle das pragas do amendoim. Informe Agropecuário, v.7, n.82, p.67-71, 1981.

WAQUIL, J.M.; VILLELA, F.M.F.; FOSTER, J.E. Resistência do milho (Zea mays L.) transgênico (Bt) à lagartadocartucho, Spodoptera frugiperda (Smith) (Lepidoptera: noctuidae). Revista Brasileira de Milho e Sorgo, v.1, n.3, p.1-11, 2002.

Recebido em 13/3/09

Aceito em $17 / 4 / 10$ 